\title{
Standing up with motor primitives
}

\author{
V. Hamburger ${ }^{1}$, K. Berns ${ }^{1}$, F. Iida ${ }^{2}$ and R. Pfeifer ${ }^{2}$ \\ ${ }^{1}$ AG Robotersysteme, TU Kaiserslautern, Germany; ${ }^{2}$ Artificial Intelligence \\ Laboratory, University of Zürich, Switzerland
}

\begin{abstract}
As observed in nature, complex locomotion can be generated based on an adequate combination of motor primitives. In this context, the paper focused on experiments which result in the development of a quality criterion for the design and analysis of motor primitives. First, the impact of different vocabularies on behavioural diversity, robustness of pre-learned behaviours and learning process is elaborated. The experiments are performed with the quadruped robot MiniDog6M for which a running and standing up behaviour is implemented. Further, a reinforcement learning approach based on Q-learning is introduced which is used to select an adequate sequence of motor primitives.
\end{abstract}

Keywords: Motor primitive, morphology, behavioural diversity, reinforcement learning, quadruped locomotion

\section{Introduction}

The theory of having a basic set of motor primitives which can be composed into a broad and general movement repertoire is an appealing organizational principle to avoid online trajectory planning. After all the representation of a function as a linear combination of less complex functions is a well established theory in mathematics and physics. Evidence in nature can be found in the human spinal cord as well as in various animals [3][4] e.g. about a dozen of non-adaptive primitives are enough to produce a frog's entire movement repertoire by means of sequence and superposition [5][6].

Motor primitives are a simple and effective approach to solving the degreeof-freedom or Bernstein problem, since fixed motor primitives significantly reduces the search space of possible postures and trajectories.

Moreover, it is widely believed that all sorts of movements seem to be designed as cyclic motions. Analysis of animal locomotion suggests that these 
motions are generated by neural networks which are capable of generating basic rhythmic motor activity [8].

These concepts motivated us to create a methodology that helps to derive several design principles for cyclic motor primitives. Questions which have to be answered are how can a basic set of fixed primitives, which is called vocabulary, be derived from morphological properties and how it affects behavioural diversity, robustness and learning process. Robustness in this context means that behaviours are tolerant against changes in morphology, environment and posture. Further, abstract, task and platform independent measures are provided to categorise and evaluate single motor primitives, entire vocabularies and behavioural diversity.

This methodology is applied to the quadruped machine MiniDog6M, which can be seen in Figure 1, for running and standing up behaviour.

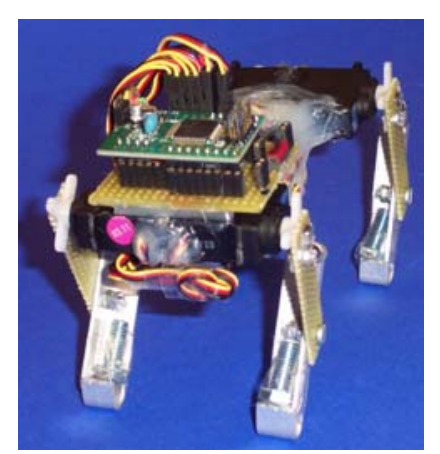

Fig. 1. MiniDog6M

Fast running is obtained by a minimalistic biologically inspired design which means that most of the control is compensated by exploiting some simple physics, such as the resilient properties of a spring yielding a passive degree of freedom in each leg. The six motors (servos in shoulders, hips and spine) are controlled by a simple sinusoidal function without having to differentiate stance and flight phases. Physically realistic simulations of MiniDog6M are performed with the Open Dynamics Engine library.

\section{Classification of vocabularies}

The measures Flexibility-Index Flx and Coherence-Index Co are used to classify distinct vocabularies.

In case a motor primitive does not consider all motors, a Don't-Care term is introduced, which causes a motor to remain in its current position. This means 
that their position depends on the last motor primitive which explicitly specified and set these motors in the past. Thus the recent history of selected components must be taken into account in order to get the current angles of all six motors.

To honour this flexibility, a special Flexibility-Index Flx is introduced in Equation (1) that represents the ratio between the actual amount and the theoretically maximum of Don't-Care terms ' $x$ '.

$$
\text { Flx }=\frac{\text { actual amount of } x}{\text { max amount of } x}
$$

A second criterion for motor primitives is the inner correlation respectively homogeneity of a posture. Herefore a root posture needs to be defined, for instance home posture (here: all motors in mid position). The similarity to this root posture and the symmetry of motor positions is expressed by the Coherence-Index Co. In doing so, symmetry is regarded as primary criterion and mid position as secondary one. Symmetry in this connotation denotes parallel movement of front, hind, left and/or right hand legs.

First, the motors are grouped into "clusters of interest" attributing the fact that certain motors serve different purposes regarding their position and effective direction within the robot. For MiniDog6M we identify three groups: leg motors, bend motor, twist motor. In the special case of one motor per group (here: bend and twist motor), the goal position needs to be compared to the root posture only.

Tab. 1. Coherence distribution for each cluster (Bend, Twist, Legs)

\begin{tabular}{|c|c|c|c|c|c|c|c|c|}
\hline Bend/Twist & & 0 & & $\mathrm{x}$ & $+/-$ & & & \\
\hline$C o($ Bend $/ \mathrm{T}$ & ist) & 1.0 & & 33 & 0.0 & & & \\
\hline legs & $\begin{array}{l}0 \\
0\end{array}$ & & & $\left(\begin{array}{l}0 \\
0\end{array}\right)$ & $\begin{array}{l}A- \\
+\lambda-\end{array}$ & $\left(\begin{array}{l}0 \\
0\end{array}\right) *$ & $\left(\begin{array}{l}* \\
*\end{array}\right)$ & $\begin{array}{lll}x & x \\
x & x\end{array}$ \\
\hline Co(legs) & 1. & & & 0.66 & 0.5 & 0.333 & 0.167 & 0 \\
\hline
\end{tabular}

The tables in Table 1 list the coherence distribution for each cluster. '0' stands for mid position (high correlation), 'x' Don't-Care (correlation estimated with the probability of mid position) and ' $*$ ' stands for minimum or maximum (low correlation). The circle denotes parallel movement. Note that the orientation of the robot is not given, so the head may be set on either side. Hence, an encircled pair of legs can be deemed front, hind, left or right hand, without loss of generality. 
Merging the Coherence-Index of spine and legs, we weight the corresponding values in ratio of the number of motors as shown in Equation (2).

$$
\text { Co }(\text { motor primitive })=\frac{1}{n} \cdot \sum_{\text {all groups }} \operatorname{Co}(\text { group }) \cdot n_{\text {grp }}
$$

The Coherence-Index of an entire vocabulary as set of motor primitives is defined in Equation (3) as ratio between the summed up Coherence-Index and the theoretical maximum.

$$
\operatorname{Co}(\text { vocabulary })=\frac{\sum \operatorname{col}(\text { motor primitive })}{\max }
$$

\section{A measure for behavioural diversity}

Despite the great variety of definitions of the term intelligence, behavioural diversity is a central aspect in most of them. In order to provide a good basis of behaviours, it would be desirable to maintain a balance between diversity and heterogeneity.

In order to evaluate behavioural diversity of a given task, the BehaviouralDiversity-Index BDI can be calculated as follows:

1. Delete all sequences that consist of another (shorter) sequence plus some prefix. (It is always possible to lengthen a sequence by performing senseless actions beforehand.)

2. Extract groups of sequences which either have the same beginning or the same ending. Each sequence may appear in more than one group.

3. Divide every group into subgroups of identical length.

4. Calculate the diversity factor D for every group as in Equation (4).

$$
D=W_{\text {nonmbr }}(n) \cdot\left(1-\frac{n_{\text {grpmbr }}}{n}\right)+W_{\text {mbr }}(n) \cdot \sum_{\text {all groups }} \frac{n_{\text {submbr }}}{n} \cdot\left(1-\frac{n_{\text {equal }}}{l}\right)(4)
$$

$\mathrm{D}$ is a measure for the heterogenity of the corresponding group.

$\mathrm{n}_{\text {grpmbr }}, \mathrm{n}_{\text {submbr }}$ : number of group resp. subgroup members

$\mathrm{n}$ : total amount of solutions

$\mathrm{n}_{\text {equal: }}$ : number of equal steps

1: length of sequence (= number of steps)

5. Calculate BDI in Equation (5) as the product of the total amount of legal sequences with the mean diversity factor:

$$
B D I=\frac{n_{\text {total }}}{n_{\text {group }}} \cdot \sum_{\text {all groups }} D
$$


In our case study, we set $\mathrm{W}_{\text {nonmbr }}(\mathrm{n})=\mathrm{W}_{\text {mbr }}(\mathrm{n})=1$. One might argue that, under certain circumstances, equal weights are inappropriate. For these cases it is recommended to set $\mathrm{W}_{\mathrm{mbr}}(\mathrm{n})$ and $\mathrm{W}_{\text {nonmbr }}(\mathrm{n})$ as in Equations (6) and (7).

$$
\begin{aligned}
& W_{\text {mbr }}= \begin{cases}1 & n<1 \\
1+\sin \left(\frac{4 n}{\pi}\right) & 1 \leq n<2 N \\
2 & 2 N \leq n\end{cases} \\
& W_{\text {nonmbr }}= \begin{cases}2 & n<1 \\
1+\cos \left(\frac{4 n}{\pi}\right) & 1 \leq n<2 N \\
1 & 2 N \leq n\end{cases}
\end{aligned}
$$

The threshold $\mathrm{N}$ can be chosen intuitively or as average amount of sequences over all vocabularies to be compared.

\section{Experiments}

In our experiments, the robot dog will be toppled by a random force applied to its head while running. Then MiniDog6M shall get up and carry on its way. Six different vocabularies have been examined with three different shapes of the head (see Figure 2), since changes in the shape of the head have biggest impact on the agent's behaviour. (Mass and centre of mass are equal throughout all experiments.)

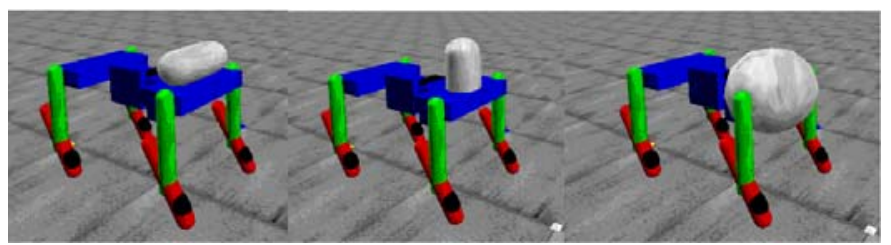

Fig. 2. Simulated versions of MiniDog6M

In order to overview the behavioural diversity, a full search algorithm was performed. These solutions have been tested on nine different ground configurations with slopes of $0^{\circ}, 22.5^{\circ}$ and $30^{\circ}$. 
Generally the support of the learning progress is an important feature of a good basic vocabulary. Here, a Reinforcement learning approach which linearly approximated a Q-Function with a Radial Basis Function network (RBF) was selected. This approach merges the advantages of both concepts. RBF has great generalisation abilities combined with optimal computational efficiency, but are highly non-linear and very difficult to analyse. Q-Learning is well formulated in mathematical terms and easy to understand for the external observer. The learning process was performed by the Reinforcement Learning Toolbox [9] with the following parameters: Epsilon-Greedy-Policy with $\varepsilon=0.3$, learning rate $\alpha=0.4$, discount factor $\gamma=0.95$ and replacing eligibility traces with $\lambda=0.9$. The reward function assigned 150 for running, 100 for standing, -1 for failure and 0 otherwise. The initial position (standing on its Head, lying on the Left, Right or Back) for each learning episode is determined by applying a random force to knock over the running dog.

\section{Results}

The vertical head cannot roll over on the back, whereas the round head cannot stand on the head or lying on the back, but rolls over on the back most easily.

Generally, Fl $>0$ results in a higher BDI, a primitive employing Don't-Care can occur in many different variants.

Depending on the chosen vocabulary, there are many examples where the solutions for one initial position are equal or a subgroup of the solutions of another initial position within the same or different head forms. The solutions for the round head intersect to a large extent with the solutions of the original head. The solutions for Right of the original head are strongly related to the ones for Left of the vertical head.

Sometimes, even symmetrical tendencies are observed. This means for the original head that all solutions for the Left are also valid for Back and about half of the solutions for Right are valid for Head. Being an implication of the form, this relation is inverted for the vertical head which means that the solutions for Back are identical to those for Right instead of Left.

Moreover, single solutions are valid for all initial positions of one head ('position stable'), some for the same initial position of different heads ('form stable'). Most surprisingly, some solutions are even valid for all heads and all initial positions (form and position stable). Depending heavily on the shape of the head, only the vertical head supports position stability for all vocabularies. An interesting fact is that sequences, that are stable in only one way, involve motor primitives with Flx $\geq 0$, but that primitives belonging to form and position stable sequences all have $\mathrm{Flx}=0$. 
On inclines, it was found, that the original and the round head perform equal or better in most cases, irrespective of the underlying vocabulary. The robustness for the vertical head can be divided into different categories. For some vocabulary the solutions for Left and Right are switched. For the second category, this is only true for either Left or Right. The other side behaves as if with the original head. After all, the original and the round heads' solutions are really robust for applications in environment with slopes of various degrees and directions. Those for the vertical head underlie a strange morphological effect that it is somehow grounded in the shape.

Comparing the results gained from learning progress and behavioural diversity, a BDI $>10$ guarantees success in not more than 100 episodes, whereas in many cases vocabularies with a lower BDI do not even reach $100 \%$ success at all. Finding a proper sequence for these configurations equals the notorious search for the proverbial needle in a haystack.

Interestingly, $\mathrm{Flx}>0$ has different consequences for different morphologies. It was expected that this type of flexibility hinders the learning process for all heads because the goal posture is no longer unambiguous. Considering the restrictions of the distinct heads, it becomes obvious that the learning progress is the faster the less initial positions are supported, but only for vocabularies with $F l x=0$. Thus the round head performed best and original head poorest. For $F l x>>0$, it is just the other way around. This insight is especially astonishing, since the solutions for round are for the most part subsets of the solutions for the original head. After all, this special type of flexibility outweighs the additional effort resulting from more initial positions. Anyway, this effect comes effortlessly if it is considered in the design phase already.

\section{Conclusion}

While it is obvious that the introduction of discrete actions alone reduces the complexity of a learning task by avoiding online trajectory planning, it was also testified that learning and control processes are closely related to the morphological properties of the executing agent. As an effect of proper or improper shape, the given task can be simplified, complicated or even be ruled out by creating situations from which it is impossible to solve the task at all. This effect can easily be exploited if considered early in the design phase. Looking at toys a robot's infrastructure can be hidden under bizarre shaped plastic covers. If designed properly the decorative shell can serve as effortless enhancement of performance.

Introducing inclines, the feasibility of the gained knowledge in changing environment without adaptation was demonstrated. This means no longer learning and still being able to succeed in new situations by making use of behavioural diversity. 
Table 2. Final ranking of vocabularies

\begin{tabular}{|c|c|c|c|c|c|c|c|c|c|c|c|c|}
\hline Vocabulary & Co & Flx & \multicolumn{3}{|c|}{$\begin{array}{c}\text { Learning } \\
\text { Progress }\end{array}$} & \multicolumn{3}{|c|}{ BDI } & \multicolumn{3}{|c|}{ Robustness } \\
\hline & & & O & V & R & O & V & R & O & V & R \\
\hline 1 & 1 & 4 & 6 & 3 & 1 & 5 & 4 & 4 & 4 & 5 & 5 \\
\hline 2 & 2 & 4 & 2 & 2 & 2 & 2 & 2 & 2 & 2 & 2 & 2 \\
\hline 3 & 5 & 4 & 5 & 4 & 4 & 6 & 6 & 6 & 6 & 4 & 4 \\
\hline 4 & 3 & 1 & 4 & 5 & 5 & 3 & 5 & 3 & 6 & 6 & 6 \\
\hline 5 & 4 & 2 & 1 & 1 & 3 & 1 & 1 & 1 & 1 & 1 & 1 \\
\hline 6 & 6 & 3 & 3 & 6 & 6 & 4 & 3 & 5 & 3 & 3 & 3 \\
\hline
\end{tabular}

The rating of vocabularies is listed in Table 2 . The numbers determine the rank ( 1 best, 6 worst) of the respective vocabulary depending on the shape of the head and the property. Co and Flx are ordered numerically (1 least, 6 highest value). $\mathrm{O}, \mathrm{V}$ and $\mathrm{R}$ stand for original, vertical and round head. The resultant ranking of the vocabulary-head-combinations leads to the assumption of a hidden system behind the qualitative results which encourages to follow up matter.

\section{References}

1. Verena Hamburger. Locomotion of a quadruped robot based on motor primitives. Diploma thesis, Robotic System Group, TU Kaiserslautern, April 2005

2. Rolf Pfeifer, Christian Scheier. Understanding Intelligence. MIT Press, 1999.

3. E. Bizzi, N. Acornero, W. Chapple, N. Hogan. Posture control and trajectory formation during arm movement. 4:2738-2744, 1984.

4. Feldman. Superposition of motor programs, I. Rhythmic forearm movement in man. 5:81-90, 1980.

5. Simon F. Giszter, Fernando A.Muss-Ivaldi, Emilio Bizzi. Convergent Force Fields Organized in the Frog's Spinal Cord. Journal of Neuroscience, Februar 1993.

6. F.A.Mussa-Ivaldi, S.F. Giszter, E. Bizzi. Linear combinations of primitives in vertebrate motor control. 1994.

7. Ansgar Büschges. Sensory Control and Organization of Neural Networks Mediating Coordination of Multisegmental Organs Organs for Locomotion. Journal of Neurophysiology, Februar 2005.

8. Gerhard Neumann. The Reinforcement Learning Toolbox, Reinforcement Learning for Optimal Control Tasks. Diploma thesis, Institute of Computer Science, TU Graz, May 2005. 